\title{
20. PALEOGENE PLANKTONIC FORAMINIFERA FROM THE WESTERN TROPICAL INDIAN OCEAN, DEEP SEA DRILLING PROJECT, LEG 24
}

\author{
Mary E. Heiman, William E. Frerichs, Department of Geology, University of Wyoming, Laramie, Wyoming \\ and \\ Edith Vincent, Department of Geological Sciences, University of Southern California, Los Angeles, California
}

\section{INTRODUCTION}

During Leg 24 of the Deep Sea Drilling Project deposits containing Paleogene planktonic foraminifera were recovered at three sites located on the Mascarene Plateau/ Central Indian Ridge/Chagos-Laccadive Ridge complex. Locations of the sites are shown in Table 1 and Figure 1.

Site 236, in 4500 meters of water, is located $270 \mathrm{~km}$ northeast of the Seychelles in the outer foothills southwest of Carlsberg Ridge. The sedimentary sequence, continuously cored, ranges from late Paleocene to Quaternary in age, with the substantial hiatuses in the Eocene and uppermost Paleocene. The Paleogene section is 127 meters thick and consists of nanno ooze to chalk, with chert in the lower 41 meters, resting on basalt. In Oligocene and upper Eocene sediments (Cores 20-28) planktonic foraminifera are rare and poorly to moderately preserved with a significant degree of fragmentation. In lower Eocene and upper Paleocene sediments (Cores 29-33) planktonic foraminifera are common to abundant but are often recrystallized.

Site 237 , in 1630 meters of water, is located on the Mascarene Plateau in a saddle joining the Seychelles and Saya de Malha bank. Coring at the site was continuous from the sea floor to 585 meters (except for one $9.5-\mathrm{m}$ joint), but from that depth to hole bottom alternate joints were cored. The site was terminated short of the basement because of operational difficulties. The section ranges in age from early Paleocene to Quaternary. The Paleogene sequence, which is 509 meters thick, includes a thick Paleocene through middle Eocene section $(481 \mathrm{~m})$ and a condensed late Eocene and Oligocene series. The sequence consists of 123 meters of nanno ooze above 386 meters of nanno chalk with chert, strongly lithified in the lower part of the section, with some glauconitic horizons in the late Paleocene. Planktonic foraminifera are common to abundant and well to moderately well preserved in the Oligocene and late and middle Eocene nanno oozes (Cores 19-31). Lower Eocene and Paleocene sediments (Cores 32-67), which are lithified and partly silicified were often hard to disaggregate. Softer horizons yielded common planktonic foraminifera, but these are poorly to moderately preserved; the degree of recrystallization and silicification becomes particularly strong below Core 59 . Thin sections from indurated horizons were analyzed and are described in Chapter 21 of this volume.
TABLE 1

Location of Sites Containing Paleogene Deposits Drilled on Leg 24

\begin{tabular}{cccc}
\hline Site & Latitude & Longitude & $\begin{array}{c}\text { Water } \\
\text { Depth } \\
(\mathrm{m})\end{array}$ \\
\hline 236 & $1^{\circ} 40.62^{\prime} \mathrm{S}$ & $57^{\circ} 38.85^{\prime} \mathrm{E}$ & 4504 \\
237 & $7^{\circ} 04.99^{\prime} \mathrm{S}$ & $58^{\circ} 07.48^{\prime} \mathrm{E}$ & 1640 \\
238 & $11^{\circ} 09.21^{\prime} \mathrm{S}$ & $70^{\circ} 31.56^{\prime} \mathrm{E}$ & 2844 \\
\hline
\end{tabular}

Site 238 , in 2840 meters of water, is located near the northeast end of the Argo Fracture Zone and the adjacent southern end of the Chagos Laccadive Plateau. The continuously cored sedimentary sequence ranges in age from latest early Oligocene to Quaternary. The Oligocene section consists of 70 meters of nanno ooze resting on 29 meters of nanno chalk and volcanic ash layers above basement. Planktonic foraminifera are common to abundant throughout the Oligocene section, and are moderately well preserved; they are predominantly of small size, however, and individuals larger than $149 \mu$ are not common.

\section{PLANKTONIC FORAMINIFERAL ZONATION}

The coarse fractions $(>149 \mu)$ of samples representing 2-cm intervals were examined for their planktonic foraminiferal assemblages; species occurrences are reported in Tables 24. The planktonic foraminiferal zonation used in this report is the letter-number system developed by Banner and Blow (1965) and Blow (1969) for the late middle Eocene and Oligocene, and by Berggren (1971), as modified from Bolli (1966), for the Paleocene to middle Eocene.

\section{Site 236}

The Paleogene planktonic foraminifera at Site 236 are rare and poorly preserved. For this reason precise zonal assignments are difficult or impossible.

Core 20 contains only three species of planktonic foraminifera and these species are long-ranging forms. Core 21 contains no planktonic foraminifera. Core 22 , however, contains Globigerina angulosuturalis, Globoquadrina sellii, and Globigerina angulofficinalis. This is a P.21/N.2 assemblage and dates Cores 20 and 21 as Zone P.21/N.2.

Core 23 was not available for study and Core 24 contained only one identifiable species, Turborotalia opima 


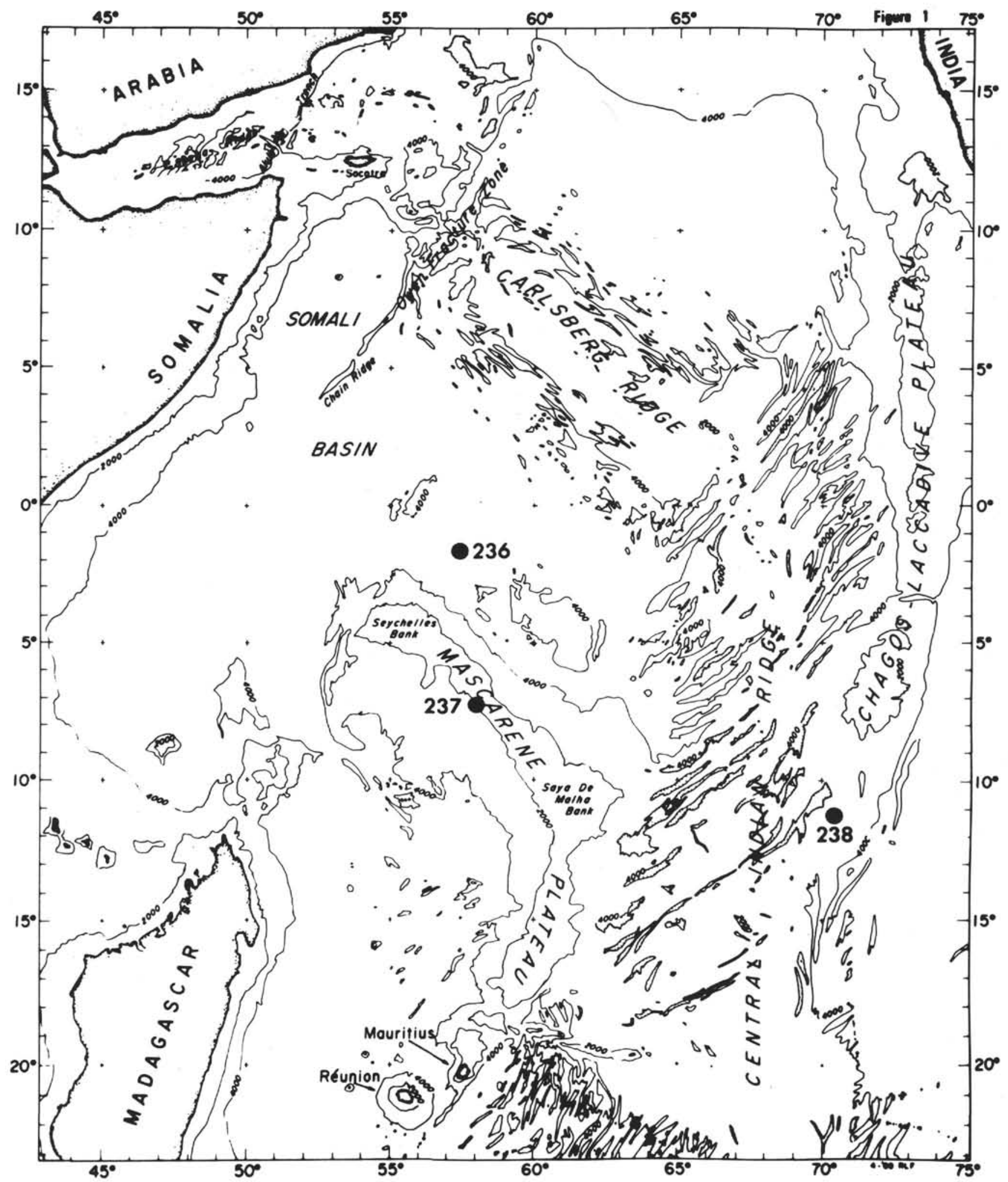

Figure 1. Location of sites containing Paleogene deposits drilled on Leg 24. 
TABLE 2

Paleogene Planktonic Foraminifera, Site 236

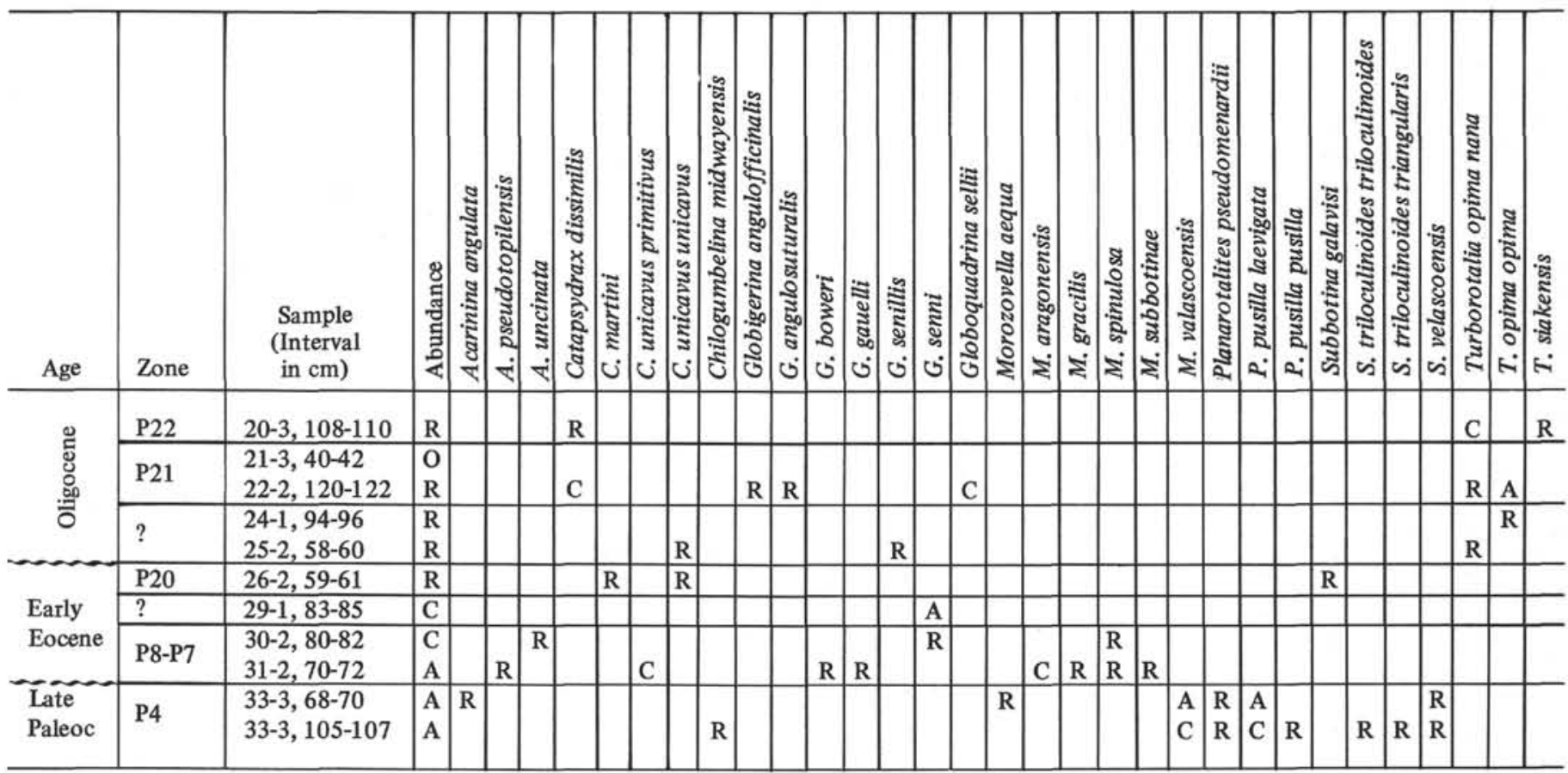

opima, which ranges from P.19 through P.21/N.2. Core 25 contains three species with concurrent ranges extending from P.16 through P.21/N.2.

Core 26 contained only three identifiable species. Included in these, however, are Catapsydrax unicavus unicavus and $C$. martini. These species occur concurrently only in Zone P.20.

Recovery in Cores 27 and 28 was very poor and this interval was not available for study; Core 29 contained only badly encrusted forms referred to as "Globigerina senni."

Core 30 also contains many encrusted forms of Morozovella spinulosa and cannot be dated with any precision. Core 31, however, contains identifiable Morozovella aragonensis and $M$. subbotinae. This assemblage is probably P.7 in age. Core 32 was not available for study.

Core 33 contains common Morozovella velascoensis and Planorotalites pseudomenardii and is assignable to Zone P.4.

\section{Site 237}

The lower part of Core 19 through Core 67 at this site contains Paleogene foraminiferal assemblages. In general, the planktonic foraminifera are quite abundant throughout this interval but preservation is highly variable and degenerates toward the base of the Paleogene. Specimens from Cores 19-23 are very well preserved. In Cores 26-53, preservation ranges from good to fair, but overall is much poorer than in Cores 19-23. Below Core 53 preservation is very poor and abundance is substantially decreased.

The bottom half of Core 19, Core 20, and the upper part of Core 21 are assigned to Zone P.22 on the co-occurrence of Globoquadrina sellii, G. tripartita, Turborotalia opima nana, and T. kugleri. The bottom half of Core 19 also contains Globigerinoides quadrilobatus immaturus, but its occurrence is thought to be due to mixing. The lower part of Core 21 is tentatively assigned to Zone P.21 as Turborotalia opima opima and $T$. siakensis are found in this part of the core and $T$. kugleri and $T$. pseudokugleri do not occur this low. The occurrence of $T$. opima opima in Core 19 is considered anomalous. Core 22 is assigned to Zone P.20 as this horizon contains the oldest occurrence of Turborotalia siakensis and the youngest occurrence of Subbotina galavisi.

Core 23 contains Globoquadrina sellii and the youngest occurrence of Pseudohastigerina and is given a Zone P.19 age. The top of Core 24 also contains Globoquadrina sellii and is assigned to Zone P.19 as well. The middle of Core 24 contains the youngest occurrence of Globigerinatheka and Truncorotaloides rohri. In addition Globoquadrina tripartita and Catapsydrax unicavus, species which first appear in Zone P.14, are found at this horizon, and Sections 3 through 5 of Core 24 are assigned to Zone P.14. A hiatus apparently exists between the samples studied in Sections 2 and 3 of Core 24.

Core 25 contains Globigerinatheka subconglobata curryi and $G$. subconglobata euganea with Catapsydrax africanus. This assemblage is mixed but a Zone P.12 age is suggested.

Core 26 is assigned to the middle Eocene P.11 Zone on the co-occurrence of Morozovella lehneri and Acarinina bullbrooki. Cores 27 through 29 are also assigned to the middle Eocene Zone P.11 as they contain Subbotina galavisi and Morozovella aragonensis together with Acarina bullbrooki in Cores 28 and 29. The youngest occurrence of Morozevella aragonensis is at the top of Core 27.

The base of Zone P.11 is placed in the lower part of Core 29 at the oldest occurrence of Globigerinatheka index, G. kugleri, and Truncorotaloides rohri. However, Subbotina galavisi and Truncorotaloides topilensis, both of which are 


\begin{tabular}{|c|c|c|c|c|c|c|c|c|c|c|c|c|c|c|c|c|c|c|c|c|c|c|c|c|c|c|c|c|c|c|c|c|c|c|c|c|c|c|c|}
\hline Age & Zon & $\begin{array}{c}\text { Sample } \\
\text { (Interval } \\
\text { in cm) }\end{array}$ & 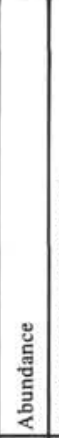 & 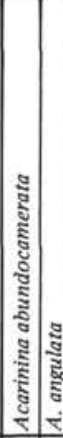 & & & & & & & & 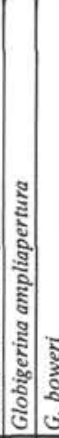 & $\mid$ & 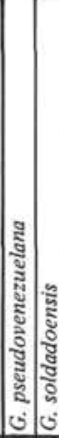 & 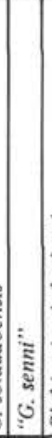 & & & & 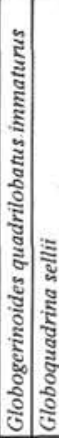 & & & & & & & & 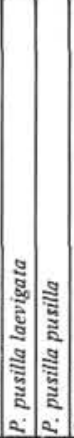 & & & & . & & & & & 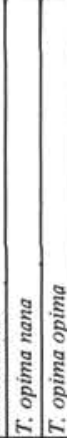 & & & $\frac{4}{4}$ \\
\hline \multirow{9}{*}{ 巳్ } & \multirow{4}{*}{$\mathrm{P}^{22}$} & $19-5,60-62$ & A & & & & & & $R$ & & & & & & & & & & \begin{tabular}{l|l}
$R$ & $R$ \\
\end{tabular} & $\mathrm{R}$ & & & & & & & & 10 & 7 & & 10 & & 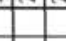 & $\mathrm{R}$ & & & & & \\
\hline & & $20-2,60-62$ & A & & & & & & $R$ & B & & & & & 1 & & & & $\mathrm{R}$ & C & & & & & & & & & 1 & & & & I & & & \begin{tabular}{l|l}
$R$ \\
\end{tabular} & & D & \\
\hline & & $20-5,60-62$ & A & & & & & & c & $\mathrm{B}$ & & & & & & & & & $R$ & c & & & & & & & & & & & & & 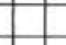 & A & A & & & \begin{tabular}{l|l}
$R$ & $R$ \\
\end{tabular} & \\
\hline & & $21-2,60-62$ & $\mathrm{~A}$ & & & & & & \begin{tabular}{l|l}
$R$ & $C$ \\
\end{tabular} & B & & & & & & & & & & c & & & & & & & & & 1 & & $\rightarrow$ & & L & $\mathrm{R}$ & & & & c & c) \\
\hline & & $21-5,60-62$ & A & & & & & & \begin{tabular}{l|l}
$R$ & $C$ \\
\end{tabular} & $\mathrm{~B}$ & & $R$ & & & $\theta$ & 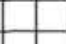 & & & $\mathrm{R}$ & c & & & & 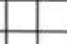 & & & & 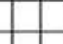 & 1 & & H & E & 11 & 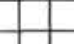 & & $\mathrm{R}$ & & $R$ & R \\
\hline & & $22-2,60-62$ & A & & & & & & \begin{tabular}{l|l}
$\mathrm{R}$ & $\mathrm{R}$ \\
\end{tabular} & & & c & & & 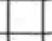 & & & & $\mathrm{R}$ & $R$ & & & & & & & & & R & & & & 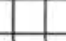 & & & & & $\mathrm{R}$ & R \\
\hline & & $23 \cdot 2,60-62$ & $\mathrm{C}$ & & & & & & \begin{tabular}{l|l}
$\mathrm{R}$ & $\mathrm{R}$ \\
\end{tabular} & & \begin{tabular}{l|l|}
$R$ & $R$ \\
\end{tabular} & $R$ & & & & & & & $\mathrm{R}$ & $R$ & & & & & & & & & \begin{tabular}{l|l}
$R$ & $R$ \\
\end{tabular} & & & & & & & & & & \\
\hline & & $23-5,60-62$ & c & & & & & & \begin{tabular}{l|l}
$R$ & $R$ \\
\end{tabular} & & $R$ & & $\mathrm{R}$ & & t & & & & & $R$ & & & & & & & & & \begin{tabular}{l|l}
$R$ & $R$ \\
\end{tabular} & & & & I & & & & & & \\
\hline & & $24-2,60-62$ & $\mathrm{C}$ & & & & & & & $\mathrm{B}$ & & $R$ & & & $\mathrm{~A}$ & & & & $\mathrm{R}$ & $R$ & & & & & & & & & & & & & a & & t & & & 1 & \\
\hline \multirow{20}{*}{ 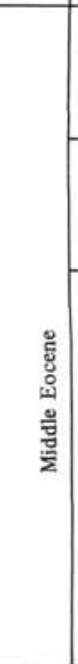 } & \multirow{4}{*}{ P14 } & $24-3,80-82$ & c & & & & & & & B & & & & & A & $\mathrm{R}$ & & & & $R$ & & & & $R$ & R & & & & 1 & & & $R$ & $R$ & & & & & 1 & \\
\hline & & $24-4,80-82$ & C & & & & & & & & & & & & A & \begin{tabular}{l|l}
$R$ & $R$ \\
\end{tabular} & & & & $R$ & & & & & & & & & $R$ & & & $R$ & $R$ & & & & & & \\
\hline & & $24-5,60-62$ & c & & & & & B & & & & & & $R$ & A & 1 & & $R$ & $\mathrm{R}$ & & & & & & & & & & & & & $R$ & $R$ & & & & & & \\
\hline & & $24-6,80-82$ & $\mathrm{C}$ & & & & & & & & & & & & A & $\begin{array}{ll}R \\
\end{array}$ & & & & & & & & $\mathrm{R}$ & $\mathrm{R}$ & & & & 1 & & & $R$ & $\mathrm{R}$ & & & & & & \\
\hline & \multirow{4}{*}{ P12 } & $25-1,80-82$ & $\mathrm{C}$ & & & $\mathrm{R}$ & & & & & & & & & 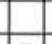 & R & $R$ & & & & & & & & & & & & & & & $R$ & & & & & & & \\
\hline & & $25-2,60-62$ & $\mathrm{C}$ & & & $\mathrm{R}$ & & $\mathrm{B}$ & & & & & & $R$ & $\mathrm{R}$ & & & $\mathrm{R}$ & & & & & $\mathrm{R}$ & & & & & & 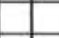 & & & $\mathrm{R}$ & & & & & & & \\
\hline & & $25-3,80-82$ & $\mathrm{C}$ & & & $R$ & & & & & & & & & $R$ & & $\mathrm{R}$ & & & & & & & & & & & $\mathrm{R}$ & & & & & & & & & & & \\
\hline & & $25-5,60-62$ & $\mathrm{C}$ & & & $R$ & & & & & & & & $R$ & c & $\mathrm{R}$ & & \begin{tabular}{|l|l|}
$R$ & $C$ \\
\end{tabular} & & & & R & & \begin{tabular}{l|l} 
\\
\end{tabular} & & & & & $R$ & & & $\mathrm{R}$ & & $\mathrm{R}$ & & & & & \\
\hline & \multirow{12}{*}{ P11 } & $26-1,110-112$ & C & & & $R$ & & & & & & & & & c & & $\mathrm{R}$ & & & & & & & & & & & & & & & a & & & & & & & \\
\hline & & $26-2,60-62$ & A & & & & & & & & & $R$ & & & C & R & $R$ & & $\mathrm{R}$ & & $\mathrm{F}$ & R & & $\mathrm{R}$ & & & & & $R$ & & & c & $\mathrm{R}$ & R & & & & & \\
\hline & & $26-4,80-82$ & $\mathrm{C}$ & & & $\mathrm{R}$ & & & & & & & & & C & & $R$ & & & & & & & & & & & & & & & & & & & & & & \\
\hline & & $27-1,80-82$ & $\mathrm{c}$ & & & & & & & & & & & & - & $\mathrm{R}$ & $R$ & & & & & $R$ & & & & & & & & & & & & & & & & & \\
\hline & & $27-2,60-62$ & $\mathrm{~A}$ & \begin{tabular}{|l|l}
$R$ \\
\end{tabular} & & & $R$ & & $\mathrm{R}$ & & & & & & $\mathrm{R}$ & $\mathrm{R}$ & $R$ & & & & & \begin{tabular}{l|l|}
$\mathrm{C}$ & $\mathrm{R}$ \\
\end{tabular} & & & & & & & $R$ & & & $\mathrm{R}$ & & $\mathrm{R}$ & & & & & \\
\hline & & $27-5,50-52$ & $\mathrm{~A}$ & $\begin{array}{lll}R & R \\
\end{array}$ & & & & & & & & $\mathrm{R}$ & & & $\mathrm{R}$ & $R$ & $R$ & & & & c & \begin{tabular}{l|l|}
$C$ & $R$ \\
\end{tabular} & & $\mathrm{R}$ & $\mathrm{R}$ & & & & $R$ & & $\mathrm{R}$ & $R$ & & & & & & & \\
\hline & & $27-6,75-77$ & $\mathrm{~A}$ & & & & & & & & & $\mathrm{R}$ & & & D & & $R$ & & & & & $R$ & & & & & & & I & & & $R$ & & & & & & & \\
\hline & & $28-1,80-82$ & $\mathrm{~A}$ & & & $\mathrm{R}$ & & & & & & & & & $\square$ & $\mathrm{R}$ & & & & & & $\mathrm{R}$ & & & $\mathrm{R}$ & & & & & & & $\mathrm{R}$ & & & & & & & \\
\hline & & $28-2,50-52$ & A & $R$ & $\mathrm{R}$ & & & & & & & $\mathrm{B}$ & & & $R$ & $\mathrm{R}$ & $R$ & & & & $\mathrm{r}$ & \begin{tabular}{|l|l|}
$\mathrm{R}$ & $\mathrm{R}$ \\
\end{tabular} & & & $\mathrm{R}$ & & & & \begin{tabular}{|l|l}
$\mathrm{R}$ & $\mathrm{R}$ \\
\end{tabular} & & $\mathrm{R}$ & \begin{tabular}{l|l}
$c$ & $R$ \\
\end{tabular} & & & & & & & \\
\hline & & $28-5,60-62$ & $\mathrm{~A}$ & $R$ & $\mathrm{R}$ & $\mathrm{R}$ & & & & & & $\mathrm{R}$ & & & $\mathrm{R}$ & $\mathrm{R}$ & $R$ & & & & \begin{tabular}{l|l}
$R$ \\
\end{tabular} & $\mathrm{R}$ & & & $\mathrm{R}$ & & & & \begin{tabular}{|l|l}
$\mathrm{R}$ & $\mathrm{R}$ \\
\end{tabular} & & 16 & \begin{tabular}{|l|l}
$\mathrm{C}$ & $\mathrm{R}$ \\
\end{tabular} & & & & & & & \\
\hline & & $29-2,116-118$ & $\mathrm{~A}$ & & & A & & & c & & & $\mathrm{R}$ & & & $\mathrm{R}$ & $\mathrm{R}$ & $R$ & & & & $\mathrm{R}$ & $\mathrm{R}$ & & & $\mathrm{R}$ & & & & \begin{tabular}{|l|l} 
& $R$ \\
\end{tabular} & & C & \begin{tabular}{|l|l}
$R$ & $R$ \\
\end{tabular} & & & & & & & \\
\hline & & \begin{tabular}{|l|}
$29-5,60-62$ \\
\end{tabular} & A & & $\mathrm{R}$ & $\mathrm{R}$ & & & & & & & & & c| & & $R$ & & & & & $\mathrm{R}$ & $\mathrm{R}$ & & \begin{tabular}{l|l} 
R \\
\end{tabular} & & & & \begin{tabular}{|l|l} 
& $R$ \\
\end{tabular} & & & \begin{tabular}{|l|l}
$R$ & $R$ \\
\end{tabular} & & & & & & & \\
\hline
\end{tabular}


TABLE 3 - Continued

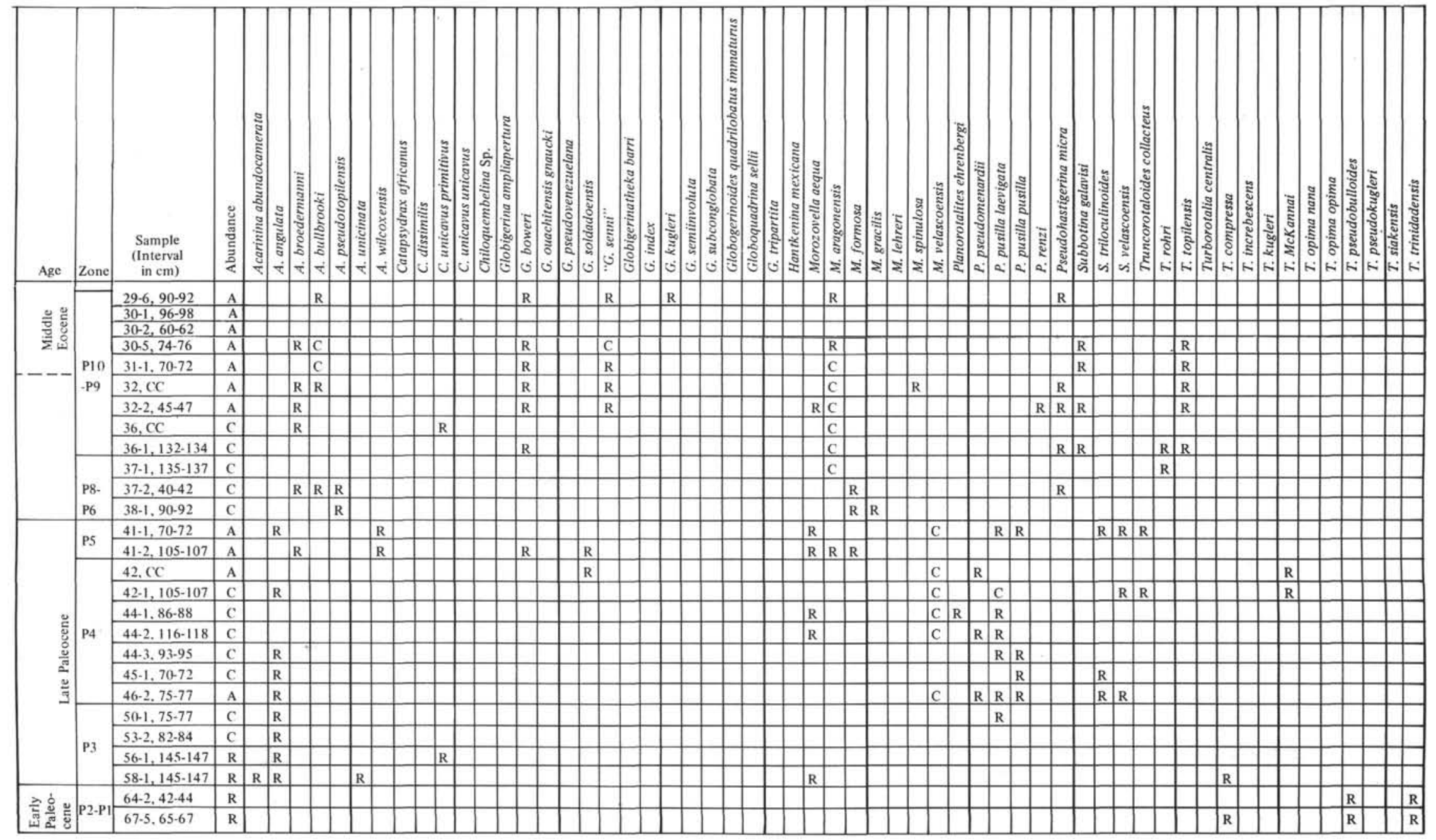


TABLE 4

Paleogene Planktonic Foraminifera, Site 238

\begin{tabular}{|c|c|c|c|c|c|c|c|c|c|c|c|c|c|c|c|c|c|c|c|c|c|c|c|c|}
\hline Age & Zone & $\begin{array}{l}\text { Sample } \\
\text { (Interval } \\
\text { in cm) }\end{array}$ & 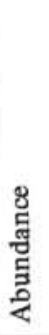 & 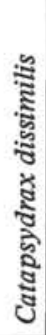 & 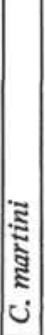 & 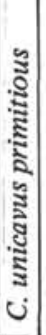 & 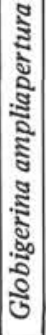 & 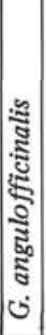 & 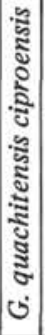 & 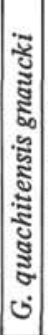 & 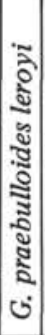 & 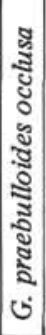 & 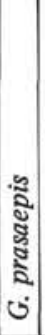 & 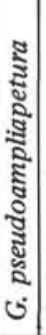 & 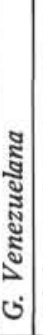 & 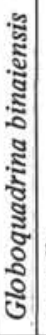 & : & 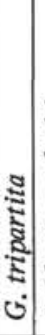 & 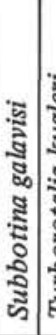 & 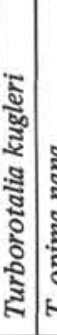 & 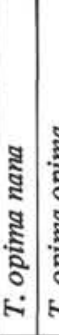 & 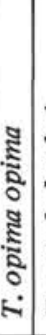 & 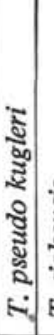 & 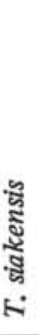 \\
\hline \multirow{4}{*}{ 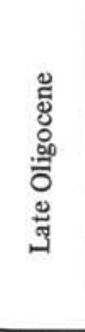 } & $\mathbf{P} 22$ & $\begin{array}{l}41-3,116-118 \\
48-2,123-125\end{array}$ & $\begin{array}{l}\mathrm{C} \\
\mathrm{C}\end{array}$ & $\mathrm{R}$ & & & & & & & $\begin{array}{l}\mathrm{R} \\
\mathrm{R}\end{array}$ & & $\mathbf{R}$ & & $\begin{array}{l}R \\
R\end{array}$ & $\begin{array}{l}R \\
R\end{array}$ & $\mathrm{R}$ & $\begin{array}{l}\mathrm{C} \\
\mathrm{R}\end{array}$ & $\begin{array}{l}\mathrm{R} \\
\mathrm{R}\end{array}$ & $\begin{array}{l}\mathrm{R} \\
\mathrm{R}\end{array}$ & & $\mathrm{R}$ & $\begin{array}{ll}C & 1 \\
R & \end{array}$ & $\begin{array}{l}R \\
R\end{array}$ \\
\hline & & $49-2,82-84$ & 0 & & & & & & & & & & & & & & & & & & & & & \\
\hline & P22-P21 & $50-2,80-82$ & $\mathrm{R}$ & & & & & & & $\mathrm{R}$ & $\mathrm{R}$ & $\mathbf{R}$ & $\mathrm{R}$ & & & $\mathrm{R}$ & $\mathrm{R}$ & $\mathrm{R}$ & $\mathrm{R}$ & & & $\mathrm{R}$ & \begin{tabular}{l|l}
$\mathbf{R}$ & \\
\end{tabular} & $\bar{C}$ \\
\hline & $\mathbf{P} 20$ & $\begin{array}{l}51-2,93-95 \\
52-2,67-69 \\
52-5,85-87 \\
53-2,125-127 \\
53-5,110-112\end{array}$ & $\begin{array}{l}\mathrm{C} \\
\mathrm{C} \\
\mathrm{C} \\
\mathrm{C} \\
\mathrm{R}\end{array}$ & $\begin{array}{l}\mathrm{R} \\
\mathrm{R}\end{array}$ & \begin{tabular}{|l|}
$\mathrm{R}$ \\
$\mathrm{R}$ \\
$\mathrm{R}$ \\
$\mathrm{R}$
\end{tabular} & $\mathrm{R}$ & R & & & & \begin{tabular}{|l|}
$\mathrm{R}$ \\
$\mathrm{R}$
\end{tabular} & & $\begin{array}{l}\mathrm{R} \\
\mathrm{R} \\
\mathrm{R} \\
\mathrm{R}\end{array}$ & & & $\begin{array}{l}\mathrm{R} \\
\mathrm{R}\end{array}$ & \begin{tabular}{|l|}
$\mathrm{R}$ \\
$\mathrm{R}$ \\
$\mathrm{R}$ \\
$\mathrm{R}$
\end{tabular} & \begin{tabular}{l|}
$\mathrm{C}$ \\
$\mathrm{A}$ \\
$\mathrm{C}$ \\
$\mathrm{C}$
\end{tabular} & $\begin{array}{l}\mathrm{R} \\
\mathrm{R}\end{array}$ & & \begin{tabular}{l|l}
$R$ & \\
$R$ & \\
$R$ &
\end{tabular} & $\begin{array}{l}\mathrm{R} \\
\mathrm{C} \\
\mathrm{C}\end{array}$ & & \\
\hline $\begin{array}{l}\text { Early } \\
\text { Oligo }\end{array}$ & P19 & $54-1,126-128$ & $\mathrm{C}$ & & & & $\mathbf{R}$ & C & $\mathrm{R}$ & $\mathrm{R}$ & $\mathrm{R}$ & & & $\mathrm{R}$ & & & $\mathbf{R}$ & $\mathbf{R}$ & & & & & & \\
\hline
\end{tabular}

known to range not lower than P.11, are present in Cores 30 through 36 . The presence of these two species in this interval is thought to be due to mixing, and this interval is tentatively assigned to Zones P.10/P.9. The oldest appearance of Hantkenina, in the upper part of Core 30, may indicate the base of Zone P.10; however, Zones P.10 and P.9 were not differentiated. The early/middle Eocene boundary at the site was drawn within this interval, between Cores 31 and 32, on the basis of nannofossil and radiolarian data. This position is consistent with a constant sedimentation rate throughout early and middle Eocene times.

Mixing is evident in the interval between Cores 37 and 41, and Zones P. 8 through P.6 were not differentiated. It is possible, however, that the base of the common and persistent occurrence of Morozovella aragonensis in Core 37, Section 1 marks the base of Zone P.7. The top of Zone P.5 is placed in Core 41, Section 1 at the youngest occurrence of Morozovella velascoensis; Section 2 of Core 41 is also assigned to Zone P.5 and rare occurrences of $M$. aragonensis and $M$. formosa at this level are thought to be due to displacement.

Planktonic assemblages in Cores 42,44 , and 46 contain Planorotalites pseudomenardii and Cores 42 through 46 are assigned to Zone P.4. Some Cretaceous elements were noted in this zone.

Cores 47 through 49 are indurated and did not yield sediments soft enough to be disaggregated. The fauna in Cores 50 through 67 was quite sparse, and below Core 53 preservation was very poor.

Cores 50 through 58 contain Acarinina angulata, but lack Morozovella velascoensis and Planorotalites pseudomenardii. Core 58 also contains specimens of Acarinina abundocamerata, A. uncinata, and Turborotalia compressa. Concurrent ranges for these species indicate a zonal age of P.3.
The only species observed in Core 64 were Turborotalia pseudobulloides and $T$. trinidadensis. Core 67 contains these two species and also specimens of Turborotalia compressa. The absence of Acarinina abundocamerata and $A$. angulata indicates that these cores are older than P.3, but it is impossible to determine whether they should be assigned to Zone P.1 or P.2.

\section{Site 238}

The Paleogene planktonic foraminifera at Site 238 are neither abundant nor diverse, and preservation is poor to fair. Consequently, zonal assignments are not always as precise as desired.

Cores 47 and 48 are assigned to Zone P.22/N.3 as Turborotalia kugleri is found in each core, and no early Miocene species are associated with it. Core 49 was barren of planktonic foraminifera. Core 50 contains Turborotalia pseudokugleri and Globoquadrina binaiensis, abundant in Core 47 , but only questionably identified in Core 50 , which indicates a P.22/N.3 age. The same core, however, contains Globigerina prasaepis and Turborotalia opima opima, species which may range as high as Zone P.22/N.3. Some mixing is probable and a definite zonal age cannot be applied. Mixing was apparent as well in Cores 47 and 48, as Subbotina galavisi was found in each core as well as in Core 50 .

Core 51 contains the youngest occurrences of Catapsydrax unicavus primitivus and C. martini. These forms first appear in Zone P.20, the age assigned here.

Cores 52 through 53 can also be assigned to Zone P. 20 due to the absence of the genus Pseudohastigerina. The lowermost core, Core 54, however, contains rare Globigerina pseudoampliapertura, a species which becomes extinct in Zone P.19. Pseudohastigerina was not noted in the sample either, but the assemblage is tentatively assigned to Zone P.19. 


\section{REFERENCES}

Banner, F. T. and Blow, W. H., 1965. Progress in the planktonic foraminiferal biostratigraphy of the Neogene: Nature, v. 208, p. 1164-1166.

Berggren, W. A., 1971. Tertiary boundaries and correlations. In Funnell, B. F. and Riedel, W. R. (Eds), Micro- palaeontology of the oceans: Cambridge (Cambridge Univ. Press), p. 693-809.

Blow, W. H., 1969. Late Middle Eocene to Recent planktonic foraminiferal biostratigraphy: Internatl. Conf. Plankt. Microfossils Proc. 1st, p. 199-422.

Bolli, H. M., 1966. Zonation of Cretaceous to Pliocene marine sediments based on planktonic foraminifera: Venez. Geol. Min. Petrol, Bol. Inform., v. 9, p. 3-32. 